NISTIR 8258

\title{
The Unit Manufacturing Process (UMP) Builder: User's Guide
}

\author{
David Lechevalier \\ William Z. Bernstein
}

This publication is available free of charge from:

https://doi.org/10.6028/NIST.IR.8258

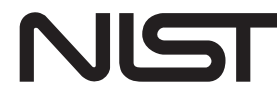

National Institute of Standards and Technology

U.S. Department of Commerce 
NISTIR 8258

\title{
The Unit Manufacturing Process (UMP) Builder: User's Guide
}

\author{
David Lechevalier \\ William Z. Bernstein \\ Systems Integration Division \\ Engineering Laboratory
}

This publication is available free of charge from:

https://doi.org/10.6028/NIST.IR.8258

July 2019

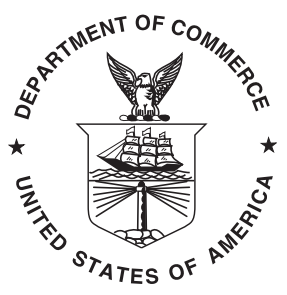

U.S. Department of Commerce

Wilbur L. Ross, Jr., Secretary

National Institute of Standards and Technology Walter Copan, NIST Director and Undersecretary of Commerce for Standards and Technology 
Certain commercial entities, equipment, or materials may be identified in this document in order to describe an experimental procedure or concept adequately. Such identification is not intended to imply recommendation or endorsement by the National Institute of Standards and Technology, nor is it intended to imply that the entities, materials, or equipment are necessarily the best available for the purpose.

National Institute of Standards and Technology Interagency or Internal Report 8258

Natl. Inst. Stand. Technol. Interag. Intern. Rep. 8258, 36 pages (July 2019)

This publication is available free of charge from: https://doi.org/10.6028/NIST.IR.8258 


\begin{abstract}
The ASTM E3012 Standard Guide for Characterizing Environmental Aspects of Manufacturing Processes provides guidelines for formally characterizing manufacturing processes. However, the difficulty that has arisen in the early use of the standard illustrates the need for intuitive tools for helping modeling experts to conform to the specified information model. The Unit Manufacturing Process (UMP) Builder was developed at the National Institute of Standards and Technology (NIST) to provide a visual interface for helping modeling experts to conform to the specified ASTM information model. The UMP Builder, a browser-based tool, integrates symbolic mathematical and guided textual inputs, helping to consistently record manufacturing process models for environmental sustainability into a repository. The Builder also serves as a curator by providing capabilities to explore and visualize models recorded in the Builder repository.

The UMP Builder is adapted from of an open source tool called the Configurable Data Curation System (CDCS) developed at NIST. The CDCS was originally developed to capture, share, and transform materials data, allowing users to upload an XML schema and create instances of that schema. In the UMP Builder, the schema used for creating the models is frozen. The schema follows the guidelines from the ASTM E3012 standard. The Builder also provides unique features that are specific in order to facilitate the modelling of UMPs. This user's guide aims to provide guidelines to use the UMP Builder. To obtain more information (especially on the architecture), please refer to the CDCS documentation.
\end{abstract}

\title{
Key words
}

ASTM E3012; Information Modeling; Smart Manufacturing; Sustainable Manufacturing; and Unit Manufacturing Processes. 


\section{Table of Contents}

1 Introduction 1

2 User Interface $\quad 1$

2.1 Home Page 2

2.1.1 Request a New Account 2

2.1.2 Login 3

2.2 Curation 5

2.2.1 Start a New Model 5

2.2.2 Open a Work-in-Progress 6

2.2.3 Upload a Local UMP Model 7

2.2.4 Curate the Information 8

2.2.5 Curate the Meta-Information 9

2.2.6 Curate the Inputs and the Outputs 11

2.2.7 Curate the Product and Process Information 12

2.2.8 Curate the Resources 12

2.2.9 Curate the Transformations 13

2.2.10 Curate the Model Bounds 15

2.2.11 Curate the Reviews 15

$\begin{array}{ll}\text { 2.2.12 Validate and Submit the Model } & 15\end{array}$

$\begin{array}{lll}2.3 & \text { Exploration } & 16\end{array}$

2.3.1 List of the Available Models 17

2.3.2 Keyword Search through the Model List 17

$\begin{array}{lll}2.4 & \text { Visualization } & 18\end{array}$

2.4.1 Meta-Information 18

\begin{tabular}{ll}
2.4 .2 & 18 \\
\hline
\end{tabular}

2.4.3 Full List of Transformations 19

2.4.4 Additional Features 21

$\begin{array}{ll}2.5 & \text { Dashboard }\end{array}$

$\begin{array}{lll}2.5 .1 & \text { My Profile } & 21\end{array}$

2.5.2 My Submitted Models 22

$\begin{array}{ll}\text { 2.5.3 My Work-in-Progress } & 22\end{array}$

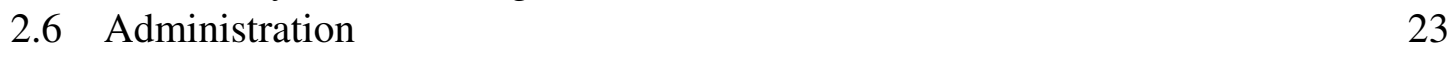

2.6.1 Manage Users 24

2.6.2 Manage Groups 25

2.6.3 Account Requests $\quad 27$

2.6.4 Contact Messages 27

3 Summary 28

References $\quad 30$ 


\section{List of Figures}

Fig. 1 Home Page as an Anonymous User 2

Fig. 2 Request a New Account 3

Fig. 3 Login 4

Fig. 4 Home Page as a Logged User 5

Fig. 5 UMP Curation 6

Fig. 6 Start a New Model 6

Fig. 7 Open a Work-in-Progress 7

Fig. 8 Open a Local UMP Model $\quad 7$

Fig. 9 Curate the Information with Tooltip Help 9

Fig. 10 Curate the Meta-information $\quad 10$

Fig. 11 Curate an Input $\quad 11$

Fig. 12 MathType Interface $\quad 12$

Fig. 13 Curate a Control Parameter 13

Fig. 14 Curate a Resource $\quad 13$

Fig. 15 Curate an Equation 14

Fig. 16 Curate a PMML Model 14

Fig. 17 Curate a Model Bound 15

Fig. 18 Curate a Review $\quad 15$

Fig. 19 Model Ready to Be Submitted 16

Fig. 20 List of Available Models $\quad 17$

Fig. 21 Result of a Keyword Search 18

Fig. 22 Visualization Page 19

Fig. 23 Additional Information $\quad 20$

Fig. 24 Profile Page 21

Fig. 25 Submitted Models Page 22

Fig. 26 Work-in-Progress Page 23

Fig. 27 User Management Page 24

Fig. 28 User List 25

Fig. 29 User Modification $\quad 26$

Fig. 30 Group List $\quad 27$

Fig. 31 Group Modification 28

Fig. 32 Account Request List 29

Fig. 33 Contact Messages $\quad 29$ 


\section{Introduction}

The ASTM E3012 Standard Guide for Characterizing Environmental Aspects of Manufacturing Processes provides guidelines for formally characterizing manufacturing processes. However, in its current form, the standard itself is not sufficient to ensure the creation and curation of UMP models that can be shared between manufacturing enterprises. Users of the standard, specifically process modelers, require computer-supported toolkits to ease the conformance to the standard data representation. In response, the UMP Builder [1] was developed at the National Institute of Standards and Technology (NIST) to provide a visual interface for helping modeling experts to conform to the specified ASTM 3012 information model. The UMP Builder, a browser-based tool, integrates symbolic mathematical and guided textual inputs, helping to consistently record manufacturing process models for environmental sustainability into a repository [2]. This document describes the features of the Unit Manufacturing Process (UMP) Builder.

The UMP Builder was developed as part of the Smart Manufacturing Systems Design and Analysis Program [3] at NIST. The tool provides a web interface to create a model, a repository to curate the models, and an interactive visualization of the models. These features provide an initial layer of governance and verification with respect to the conformance to ASTM 3012 and are widely described in this user's guide.

The UMP Builder is adapted from of an open source tool called the Configurable Data Curation System (CDCS) developed at NIST [4]. The CDCS was originally developed to capture, share, and transform materials data, allowing users to upload an XML schema and create instances of that schema. In the UMP Builder, the schema used for creating the models is frozen. The schema follows the guidelines from the ASTM E3012 standard. The Builder also provides unique features that are specific to facilitate the modelling of UMPs. This user's guide is not intended to describe the CDCS architecture or functions but to provide guidelines to use the UMP Builder's functions that might be slightly different than the CDCS functions. To obtain more information about the CDCS architecture and technology, please contact the team in charge of the CDCS or refer to the documentation ${ }^{1}$.

The remainder of this document presents the different UMP Builder features and how to use them.

\section{User Interface}

This section describes the UMP Builder user interface and its basic operations including:

- curating UMP models

- visualizing UMP models

- exploring the UMP repository

- accessing the dashboard

- administrating the UMP Builder

$\overline{{ }^{1} \text { https://www.nist.gov/itl/ssd/information-systems-group/configurable-data-curation-system-cdcs }}$ 
The home page provides the necessary access to these operations depending on the user's credentials.

\title{
2.1 Home Page
}

The home page provides generic information about the UMP Builder as shown in Figure 1.

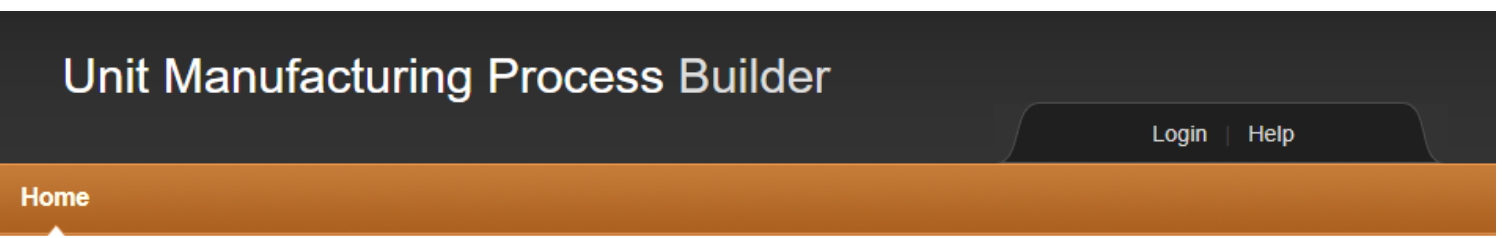

\section{Unit Manufacturing Process Builder}

\begin{abstract}
Welcome to the Unit Manufacturing Process (UMP) Builder. The UMP Builder allows researchers to record their UMP models according to the ASTM E3012-16 standard.

One of the main drivers of the UMP Builder is to support the collection of UMP models for the 2018 competition. If you are planning to participate in RAMP 2018, you can use the UMP Builder to record your model submission. The UMP Builder provides an interface for entering mathematical equations and stores each in MathML format. The UMP Builder also allows for PMML files to be included into a UMP model. After you build your UMP model, you can download it as an XML document to your local machine. If you choose to use the UMP Builder to save your models, you can submit your UMPs to the system and browse them as you please. For more information, please see the Help page.
\end{abstract}

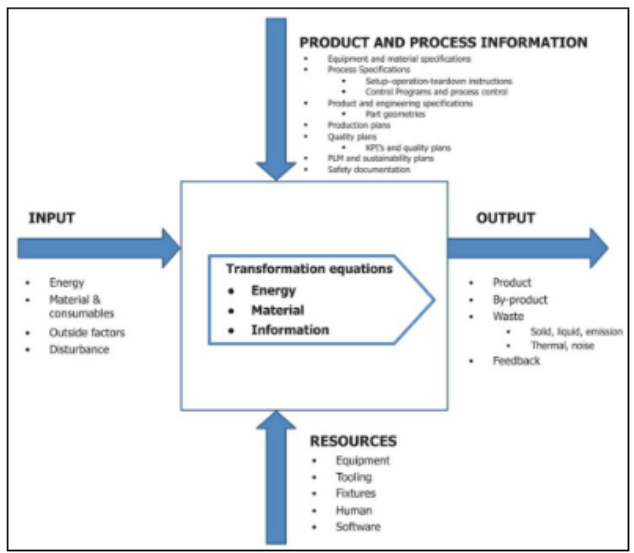

To use the tool, you must first create an account by clicking the "Login" button at the top right of the page. We welcome your suggestions and recommendations about the interface and system. Use the "Contact" button at the bottom the page to send us your comments.

Fig. 1. Home Page as an Anonymous User

As an anonymous user, you cannot access any of the feature of the tool. You first need to login or to request a new account.

\subsubsection{Request a New Account}

You first need to request a new account. Click on the login tab and click on the Request an Account button to be redirected to the required form shown in Figure 2. Provide the 
necessary information. A specific policy must be followed for the password. The password:

\section{Unit Manufacturing Process Builder}

Request New Account

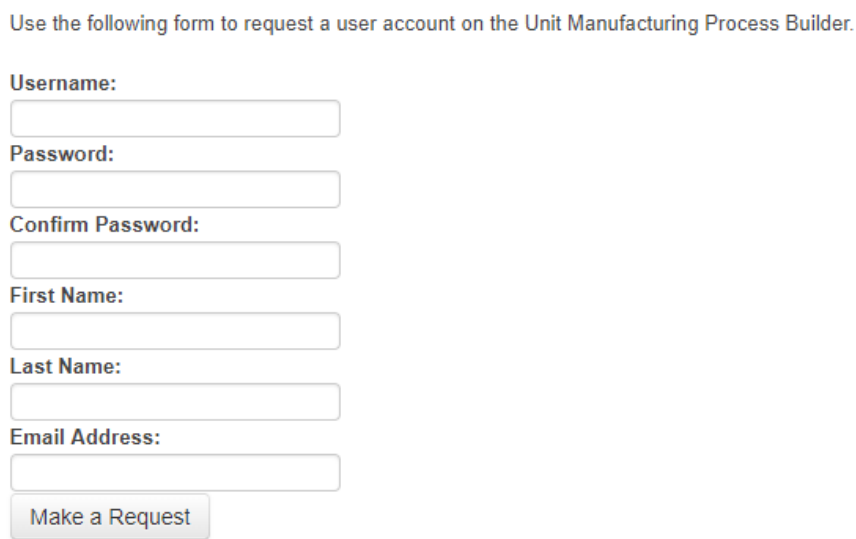

Fig. 2. Request a New Account

- must be at least 12 characters

- must contain 3 or more letters

- must contain 1 or more number

- must contain 1 or more symbol

- must contain 1 or more uppercase letter

Once requested, your account must be approved by an administrator. An email confirmation will be sent to the provided email address to confirm the request submission, and the account approval or rejection.

\subsubsection{Login}

To login, click on the Login tab at the top of the home page. You are redirected to the login page in which you can either log in or request a new account as shown in Figure 3. To login, use the credentials you have requested originally. Once logged in, the home page 


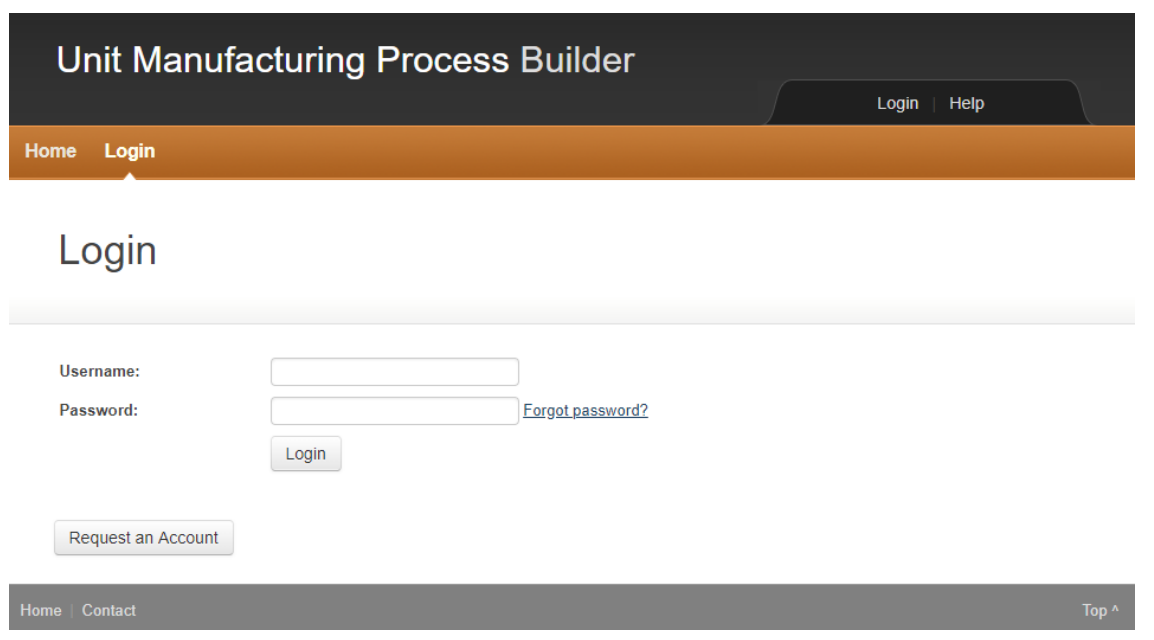

Fig. 3. Login

provides additional options, depending on the user credentials as shown in Figure 4. The available features are described in the following sections. 


\section{Unit Manufacturing Process Builder \\ Home UMP Curation UMP Exploration

Unit Manufacturing Process Builder

Welcome to the Unit Manufacturing Process (UMP) Builder. The UMP Builder allows researchers to record their UMP models according to the ASTM E3012-16 standard.

One of the main drivers of the UMP Builder is to support the collection of UMP models for the 2018 competition. If you are planning to participate in RAMP 2018, you can use the UMP Builder to record your model submission The UMP Builder provides an interface for entering mathematical equations and stores each in MathML format. The UMP Builder also allows for PMML files to be included into a UMP model. After you build your UMP model, you can download it as an XML document to your local machine. If you choose to use the UMP Builder to save your models, you can submit your UMPs to the system and browse them as you please. For more information, please see the Help page.

To use the tool, you must first create an account by clicking the "Login" button at the top right of the page. We welcome your suggestions and recommendations about the interface and system. Use the "Contact" button at the bottom the page to send us your comments.

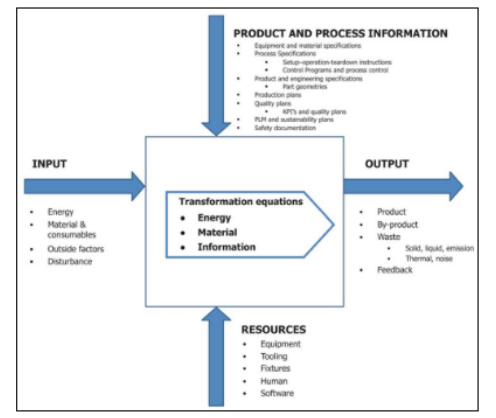

Curate your UMP

Click here to select a form template and then fill out the corresponding form

Explore the repository

Click here to search for UMP in the repository using flexible queries.

Fig. 4. Home Page as a Logged User

\subsection{Curation}

To create a new model and curate information about a UMP, you need to click on the UMP Curation tab in the home page. Three options are available as shown in Figure 5:

- Start a new UMP model

- Open a work-in-progress

- Upload a local UMP model

These options are described in the following sub-sections.

\subsubsection{Start a New Model}

When you want to start a new model in the UMP Builder, you should select the option Start a new UMP model. You will be provided with an empty form to start creating a new model. Before clicking on the Start button, you need to provide a name to your model such 


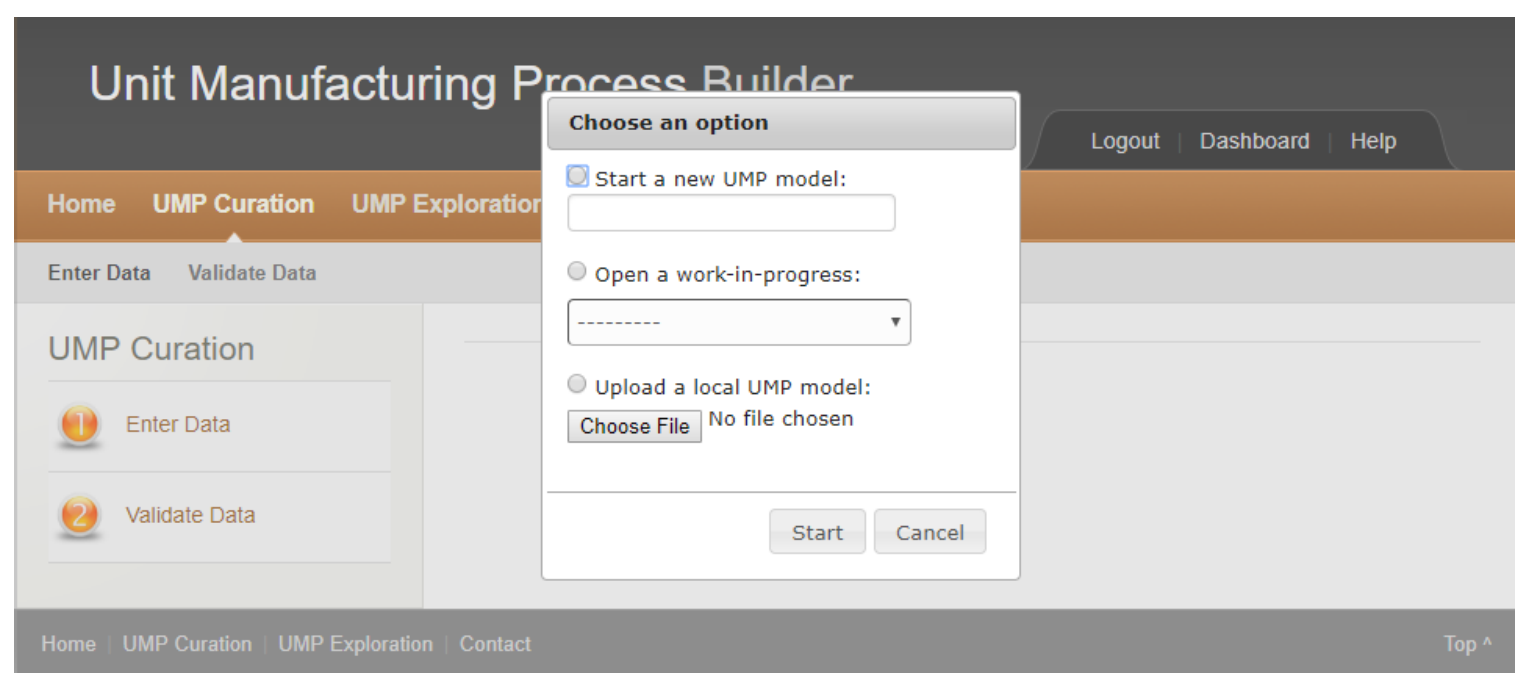

Fig. 5. UMP Curation

as "My new model" in Figure 6. Once you provided a name, you can press Start. You will be redirected to a new page with an empty form.

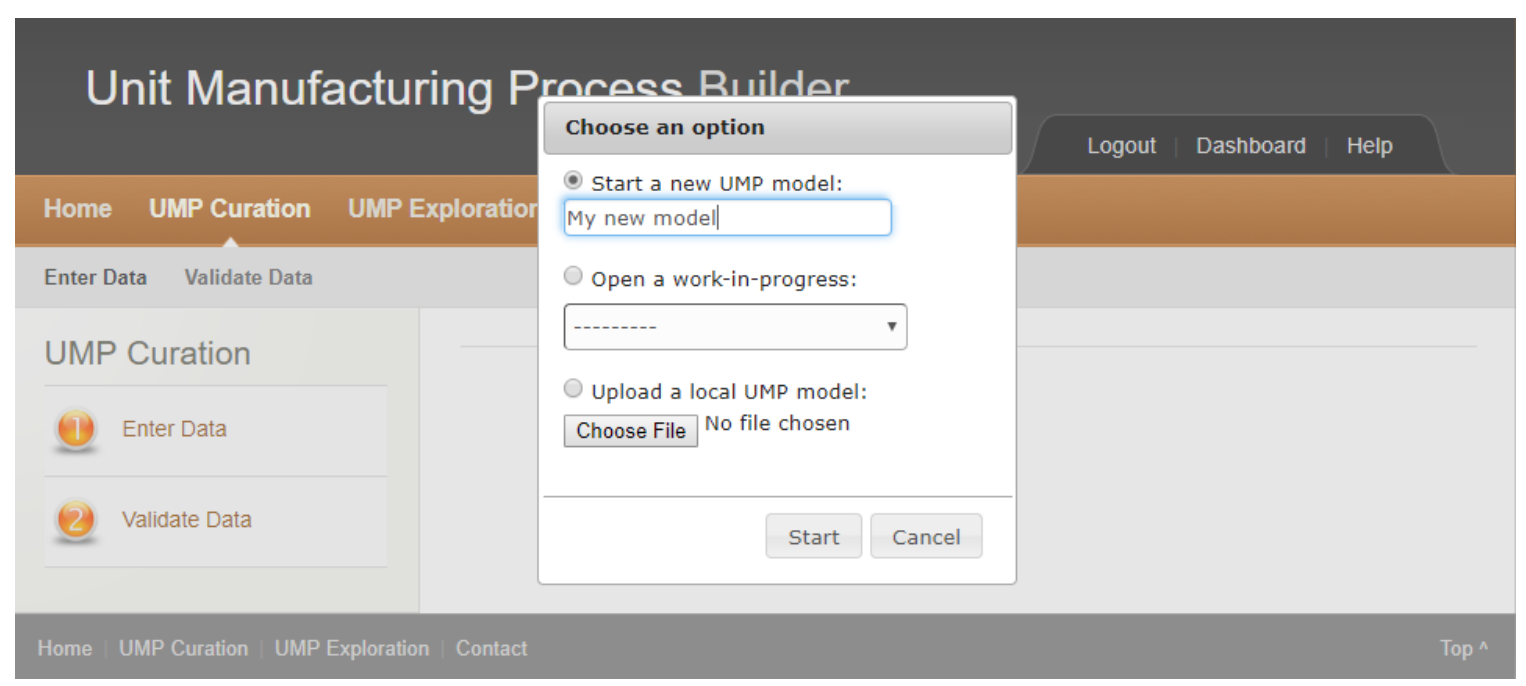

Fig. 6. Start a New Model

\subsubsection{Open a Work-in-Progress}

A model is periodically saved when you work on it. You also have the option to manually save progress. The option Open a work-in-progress allows you to reopen a model that needs additional work before being submitted to the repository. In Figure 7, you can see that "My model in progress" is available and can be reopened to provide additional information. 


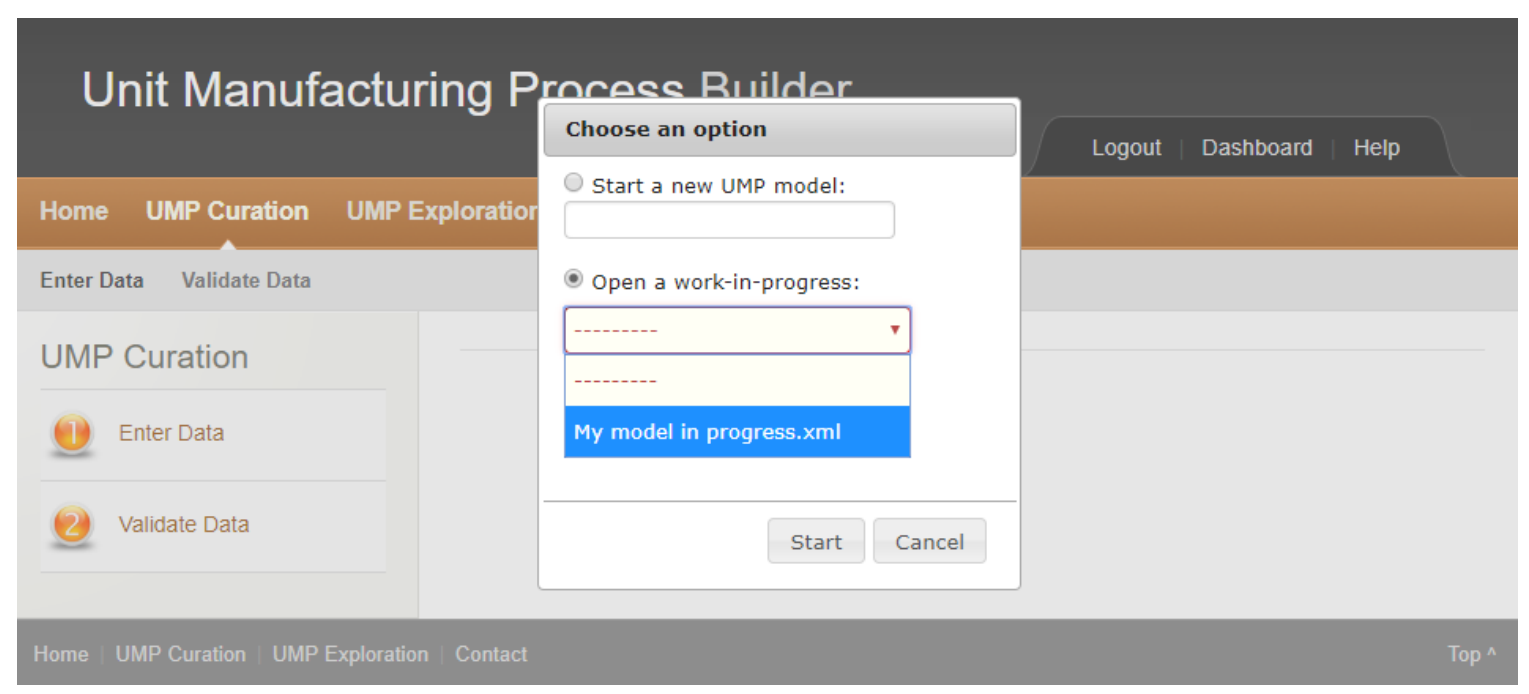

Fig. 7. Open a Work-in-Progress

\subsubsection{Upload a Local UMP Model}

The option Upload a local UMP model allows you to import a model you might have built locally with your own XML tool. The model that you import must be compliant with the schema used in the tool. The schema is available online [5]. To import your file, click on Choose file, select your UMP model and click Open in the new window shown in Figure 8, and click on start to be redirected to the new page.

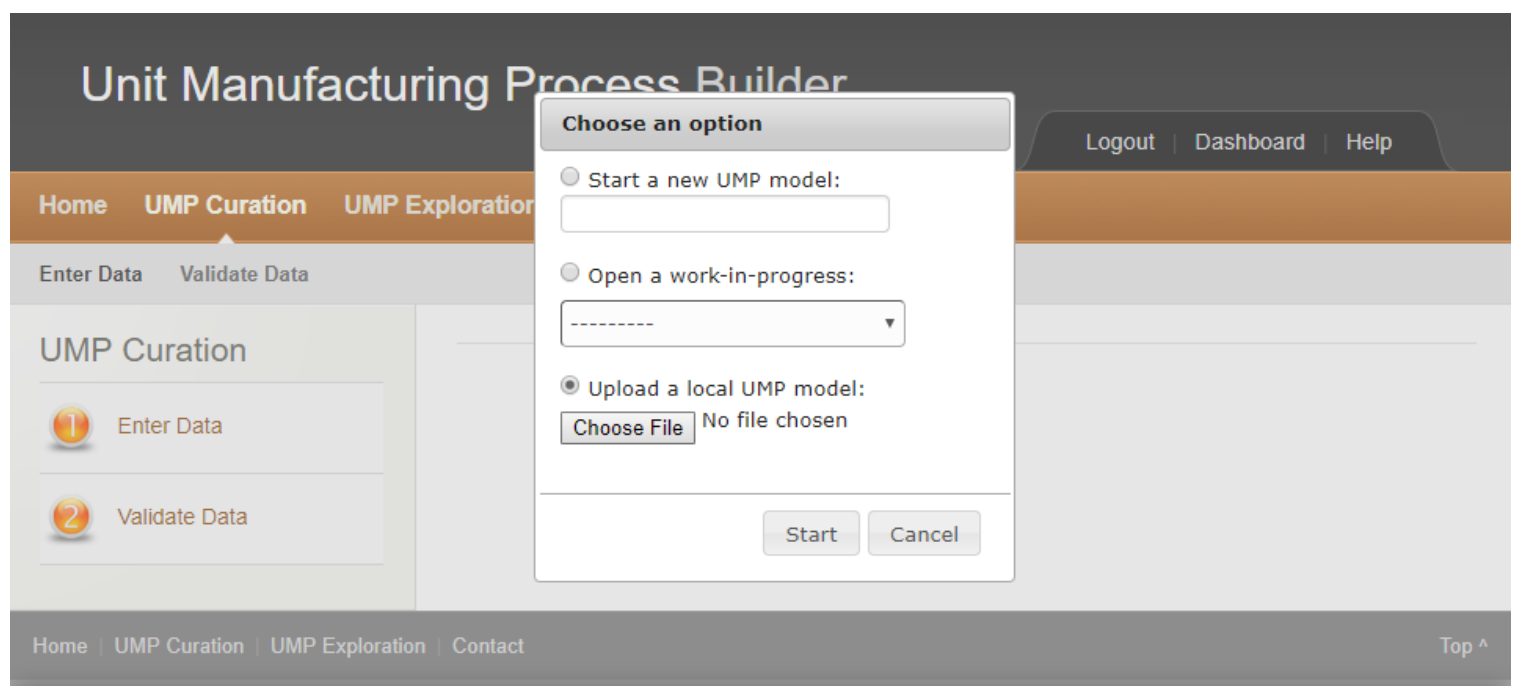

Fig. 8. Open a Local UMP Model

Once you selected one of the three options and pressed Start, you will be redirected to the new page that provides either an empty form (if you start a new model) or a form 
pre-populated with information (if you open a work-in-progress or upload a local UMP model). The new page is described in the next section.

\subsubsection{Curate the Information}

The UMP Builder provides a form to create a UMP model. Shown in Figure 9, tooltips will appear when the mouse hovers over the different fields composing the form to help you understand what kind of information is expected in the different fields.

At any moment, you can either clear all the fields, save your work-in-progress (although there is an auto-save feature included), or download the current model in an XML format. The following sub-sections will describe the different fields to build a UMP model.

In this form, you can add an attribute or an element of a given type by clicking on ${ }^{\odot}$. You can delete an attribute or an element by clicking on $\mathbf{x}$. You can expand or collapse an element by clicking on ${ }^{\oplus}$ or ${ }^{\theta}$.

Please refer to the UMP schema documentation [6] to know what type of information you should provide for each field while creating an UMP model. 


\section{Unit Manufacturing Process Builder}

\section{Home UMP Curation UMP Exploration Composer \\ Enter Data Validate Data \\ UMP Curation \\ Data Entry}

Fill out the form below to describe your UMP model. At any time, you can save your progress by clicking on "Save Work-in-Progress". Once you log back into the UMP Curation tab, you will be able to re-load your progress.

Once you have completed your UMP model, you can click "Validate Data" located on the left pane. After the model is validated according to the UMP schema, you will be able to submit your UMP model to your repository.

For every input field, a tooltip provides an explanation and examples about the required values. You can also find the full schema documentation here.

b Clear Fields $\mathbf{a}$ Save Work-in-Progress $\quad \downarrow$ Download XML

UnitManufacturingProcess

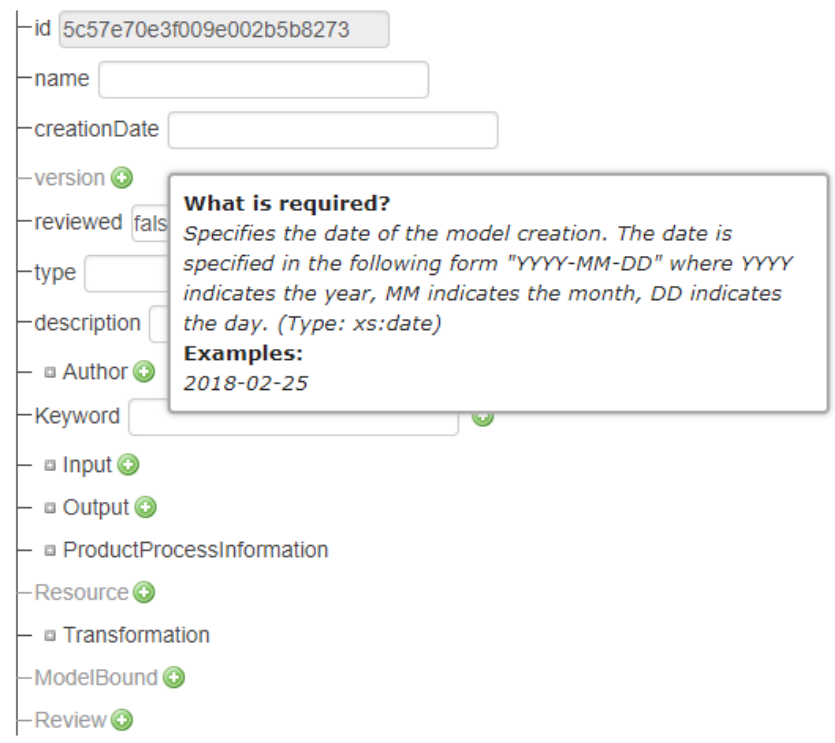

Home | UMP Curation | UMP Exploration | Composer | Contact

Fig. 9. Curate the Information with Tooltip Help

\subsubsection{Curate the Meta-Information}

The first section of the form allows you to provide model meta-information. The tooltips will provide information about the appropriate format, especially for dates. An example is provided in the Figure 10. The id field is automatically populated. 


\section{Unit Manufacturing Process Builder}

\section{Home UMP Curation UMP Exploration}

Enter Data Validate Data

UMP Curation

(1) Enter Data

(2) Validate Data

\section{Data Entry}

Fill out the form below to describe your UMP model. At any time, you can save your progress by clicking on "Save Work-in-Progress". Once you log back into the UMP Curation tab, you will be able to re-load your progress.

Once you have completed your UMP model, you can click "Validate Data" located on the left pane. After the model is validated according to the UMP schema, you will be able to submit your UMP model to your repository

For every input field, a tooltip provides an explanation and examples about the required values. You can also find the full schema documentation here.

\section{b Clear Fields \\ A Save Work-in-Progress \\ $\downarrow$ Download XML}

घ UnitManufacturingProcess

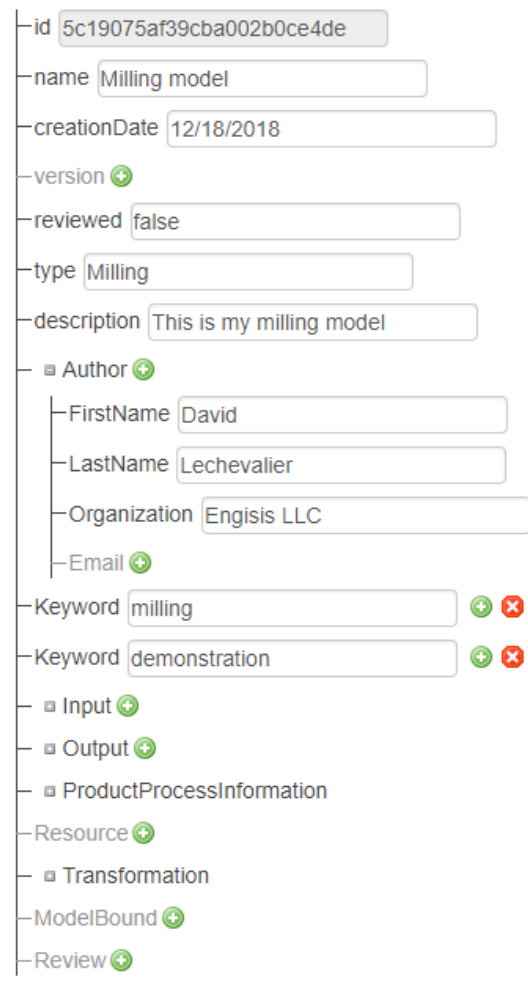

Fig. 10. Curate the Meta-information 


\subsubsection{Curate the Inputs and the Outputs}

The second and third sections of the form enables the creation of inputs and outputs. Inputs and outputs follow the same schema. An example of an Input called "Electricity" is shown in Figure 11. In a UMP model, Symbols and Bounds are always represented in a specific format called Mathematical Markup Language (MathML) [7]. To facilitate the representation of the information in this format, we used the trial version of a tool called MathType ${ }^{2}$.

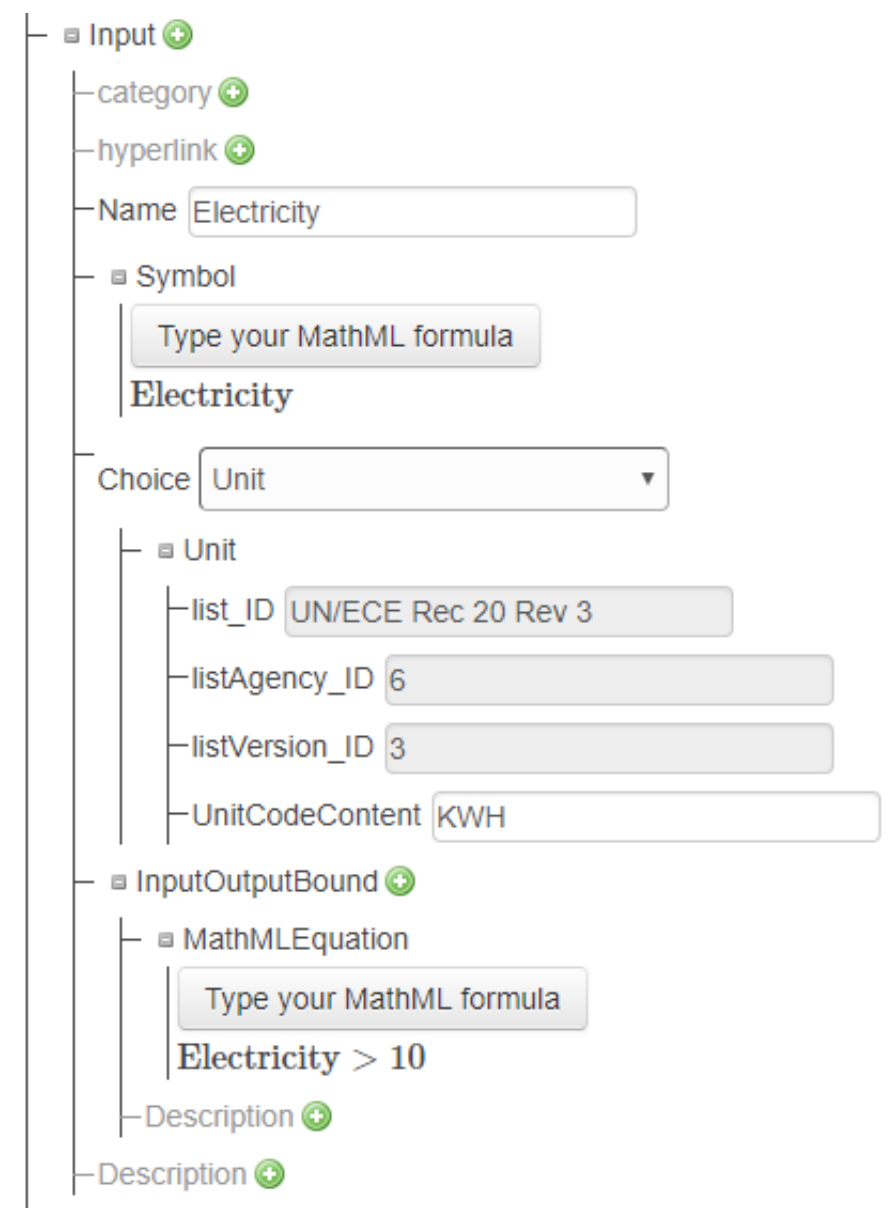

Fig. 11. Curate an Input

Figure 12 shows how the MathType interface looks like. The only requirement to properly add information in the MathML formats is to check the button TI when you add a variable name and unchecked it when you anything else. The variable name is then boxed in blue.

\footnotetext{
${ }^{2}$ http://www.wiris.com/en/mathtype
} 


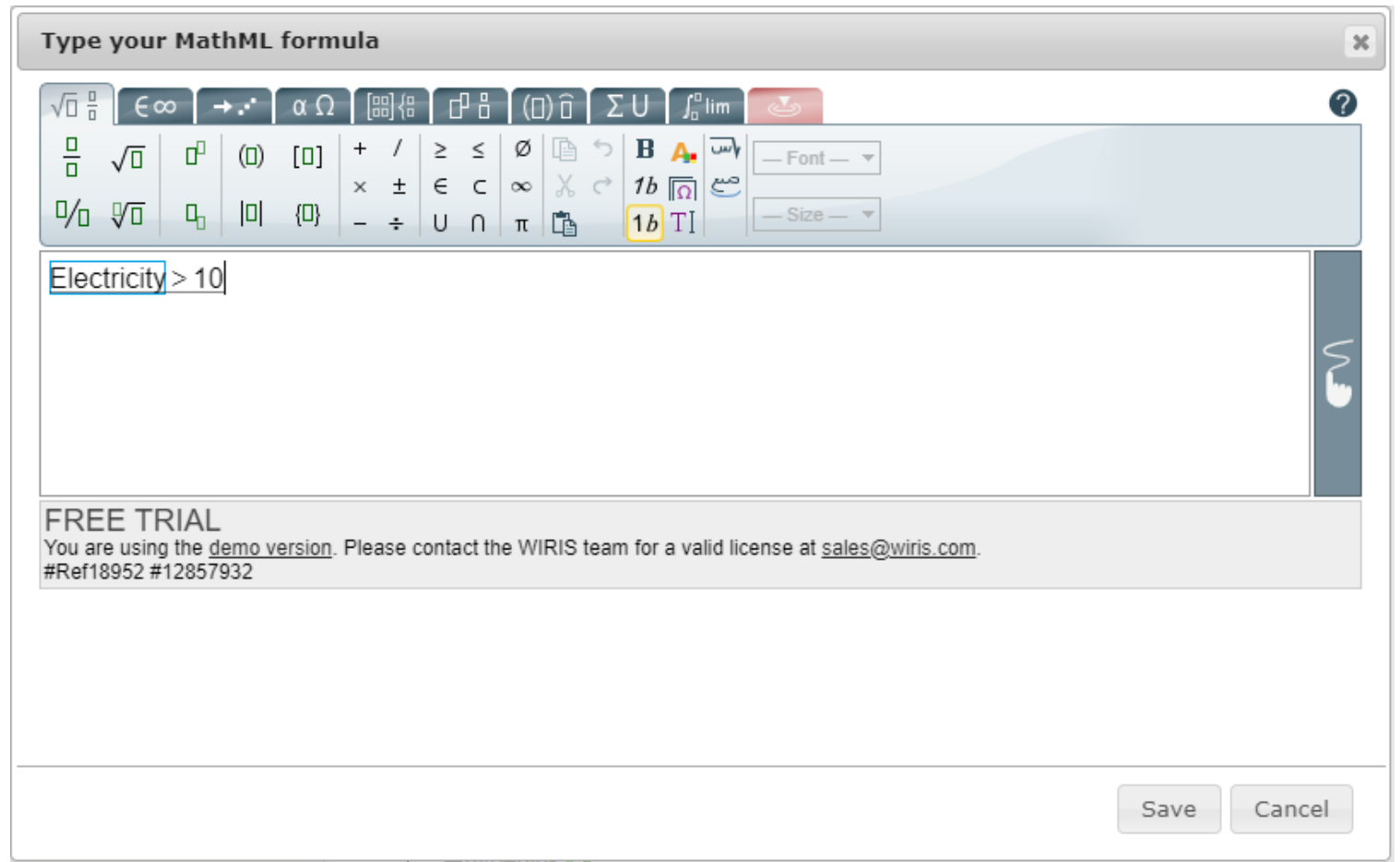

Fig. 12. MathType Interface

\subsubsection{Curate the Product and Process Information}

ProductandProcessInformation is the fourth section of the form and currently includes 5 sub sections:

- ControlParameter

- FixedParameter

- IntermediateVariable

- MetricofInterest

- SupportingInformation

ControlParameter, IntermediateVariable and MetricofInterest follow the same schema. FixedParameter includes an additional element called Value. Like the inputs and outputs, ControlParameter, FixedParameter, IntermediateVariable and MetricofInterest includes Symbols and Bounds in the MathML format. The MathType interface is also provided to include the necessary values. Figure 13 shows an example for a ControlParameter called "Depth of cut".

\subsubsection{Curate the Resources}

Resource is the fifth section of the form. Several resources can be included in the model. An example of a Resource called "Operator" is shown in Figure 14. 


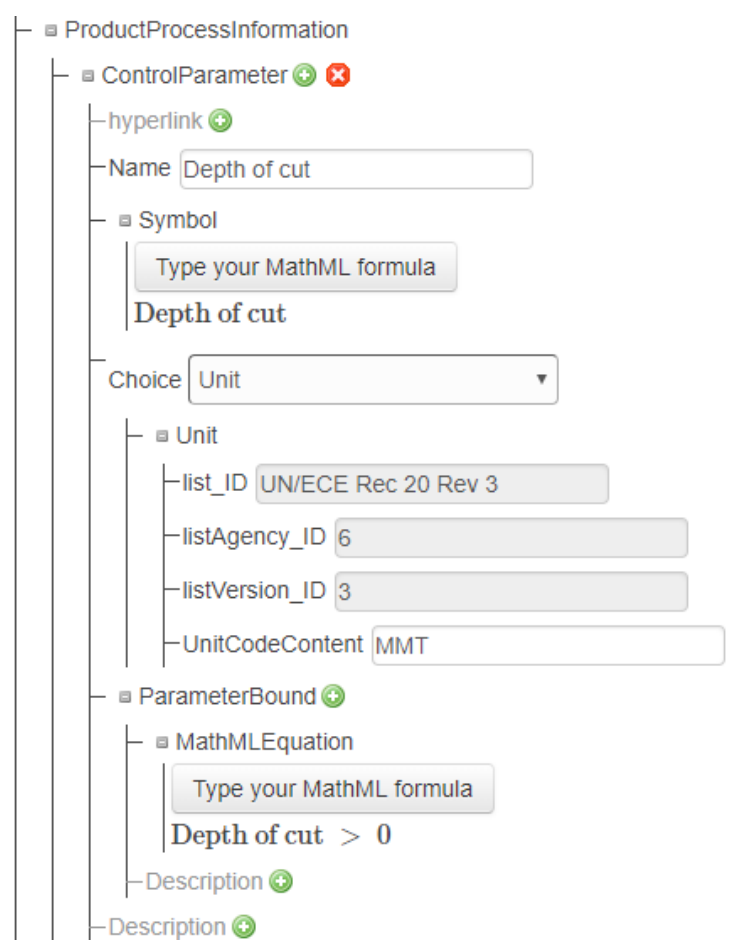

Fig. 13. Curate a Control Parameter

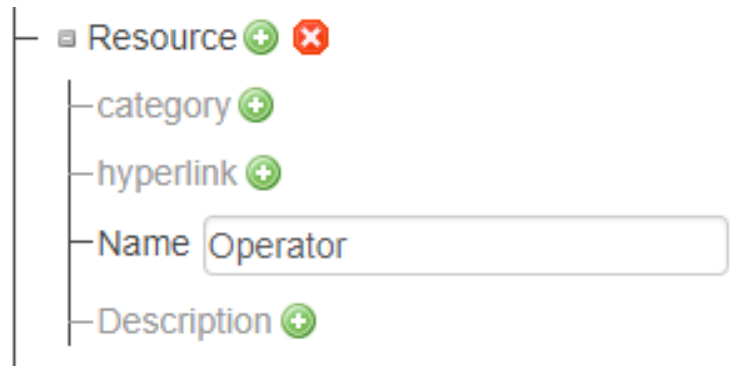

Fig. 14. Curate a Resource

\subsubsection{Curate the Transformations}

Transformation is the sixth section of the form and currently includes sub-sections:

- Equation

- PMMLModel

- FeasibilityConstraint

- Description

Equation, and FeasibilityConstraint follow the same schema. PMMLModel follows its own schema. Finally, Description provides a field to record information in a text format about the transformation represented in the model. Equation, and FeasibilityConstraint include a MathML representation. The MathType interface is also provided to include the necessary values. Figure 15 shows an example for an Equation called "Feed rate". 


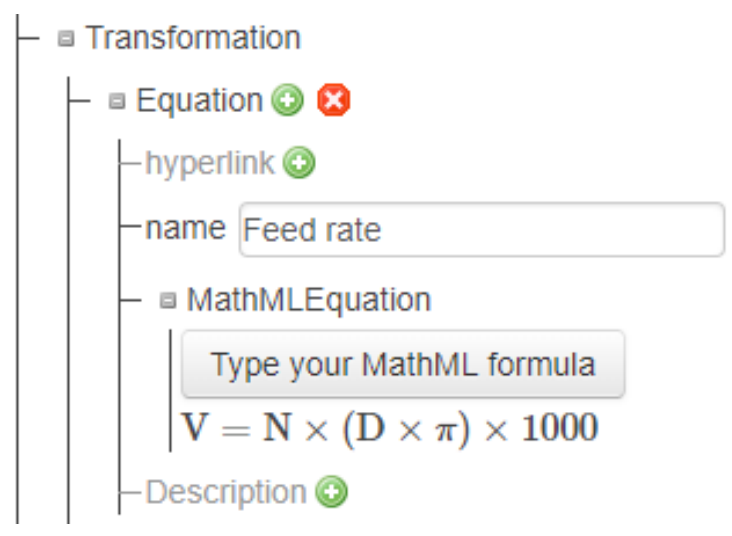

Fig. 15. Curate an Equation

A PMMLModel element is slightly different and provides the possibility to include a data-driven model in an PMML format ${ }^{3}$ instead of a MathML format. The field containing the data-driven model is automatically verifying that proper XML is added as shown in Figure 16.

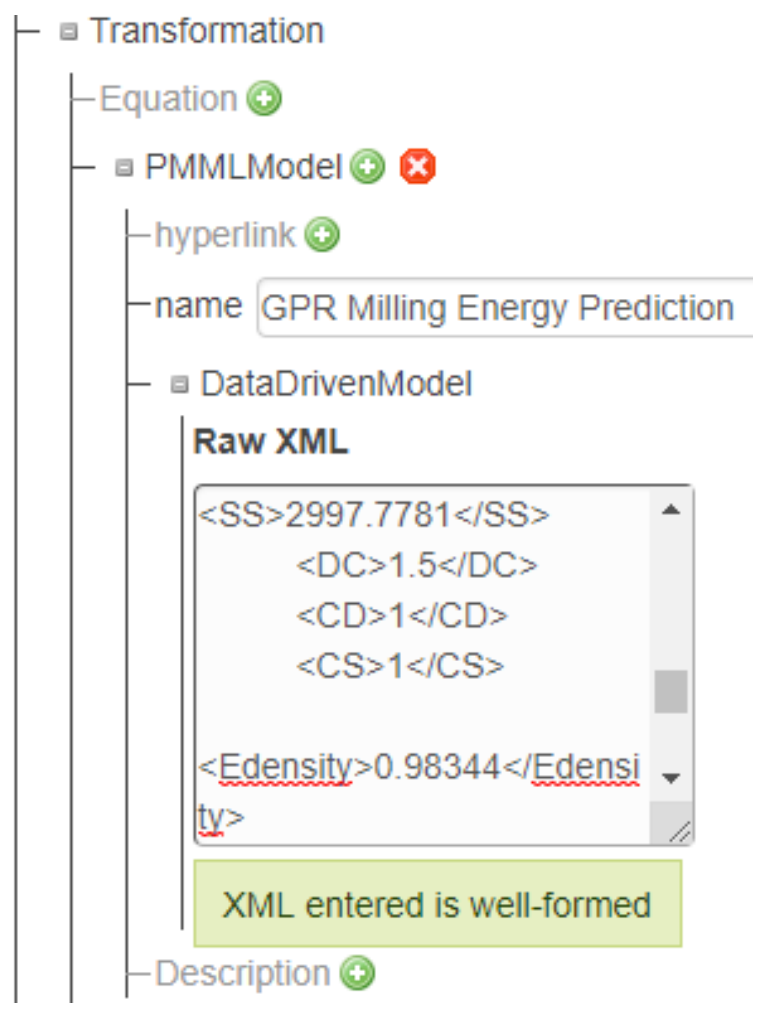

Fig. 16. Curate a PMML Model

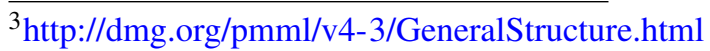




\subsubsection{Curate the Model Bounds}

A model can be valid under specific conditions. The section ModelBound enables the recording of these conditions. An example of model bound equation is provided in Figure 17. The equation is represented in the MathML format using the MathType interface.

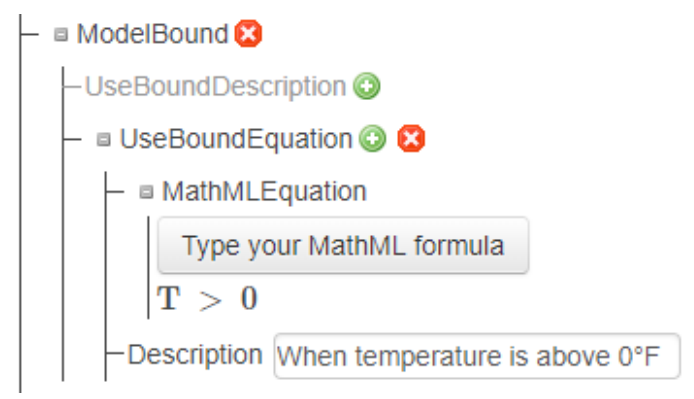

Fig. 17. Curate a Model Bound

\subsubsection{Curate the Reviews}

The last section of the forms is the Review section that enables recordings of external feedbacks about a model. An example of a Review is provided in Figure 18.

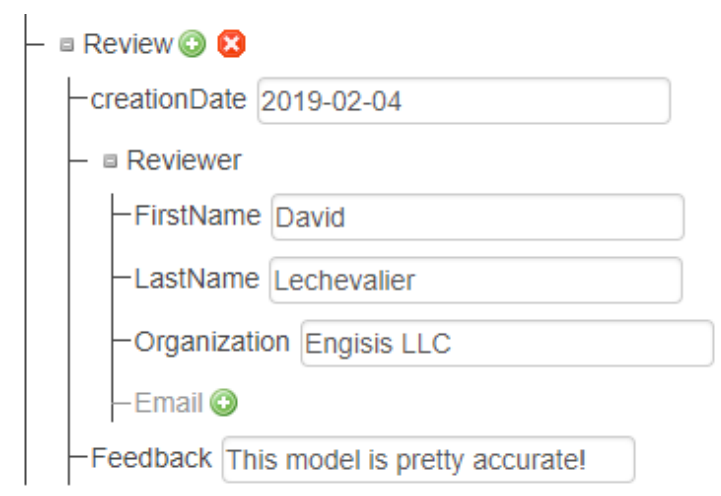

Fig. 18. Curate a Review

\subsubsection{Validate and Submit the Model}

Once you curated all the information, you can validate your model by clicking on the Validate data link on the left. This will automatically validate the information against the schema and redirect you to the visualization page if the model is correct. If the model is incorrect or values are missing, you will be notified by an error message.

You can interactively visualize your model before submitting it to the repository. Information about the visualization features are provided in Section 2.4. When you are ready to 
submit the model, click on the Submit to repository button at the top of the page. A message will confirm the submission and you will be redirected to the home page. Figure 19 shows how the visualization page looks like when the model is ready for submission.

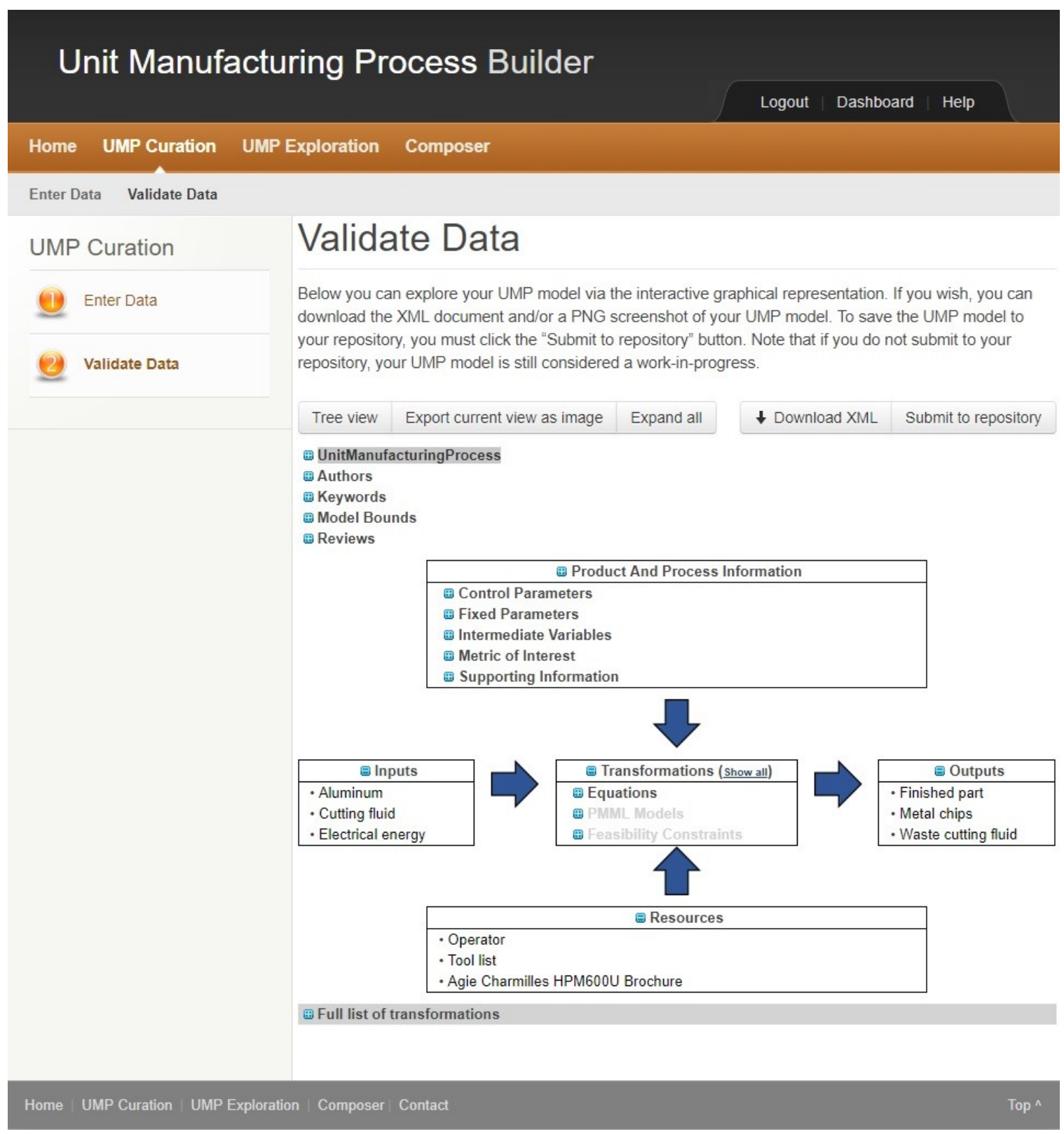

Fig. 19. Model Ready to Be Submitted

\subsection{Exploration}

To explore the UMP repository and discover the available UMP models, you need to click on the UMP Exploration tab from the home page. 


\subsubsection{List of the Available Models}

This will redirect you to a page that provides a list of all the available models as shown in Figure 20.

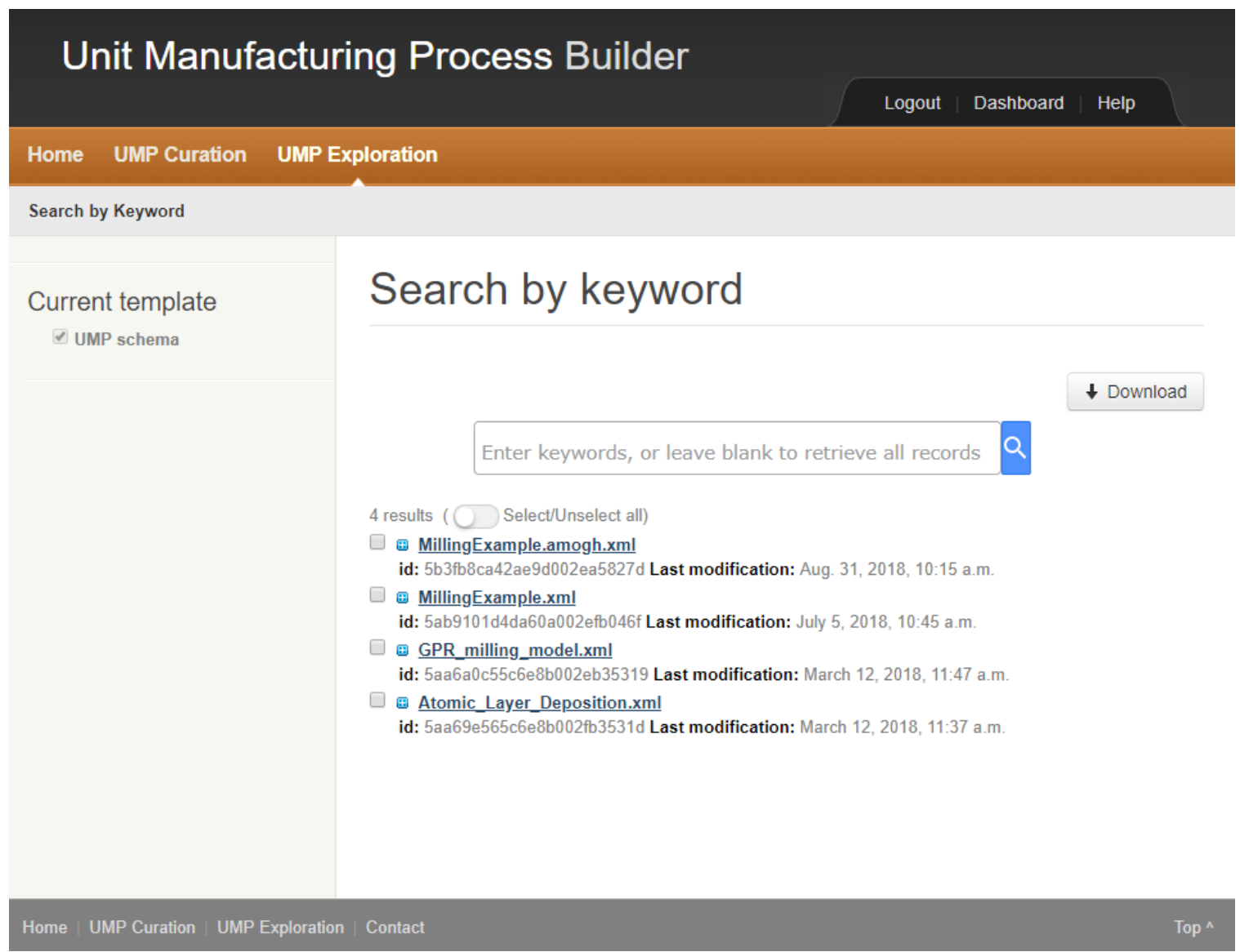

Fig. 20. List of Available Models

\subsubsection{Keyword Search through the Model List}

If you look for specific models, you can enter keywords in the input field and click on the magnifying glass button. The UMP Builder will then look for models including this keyword and refresh the list with the models matching this request. An example with the keyword "GPR" is provided in Figure 21. 


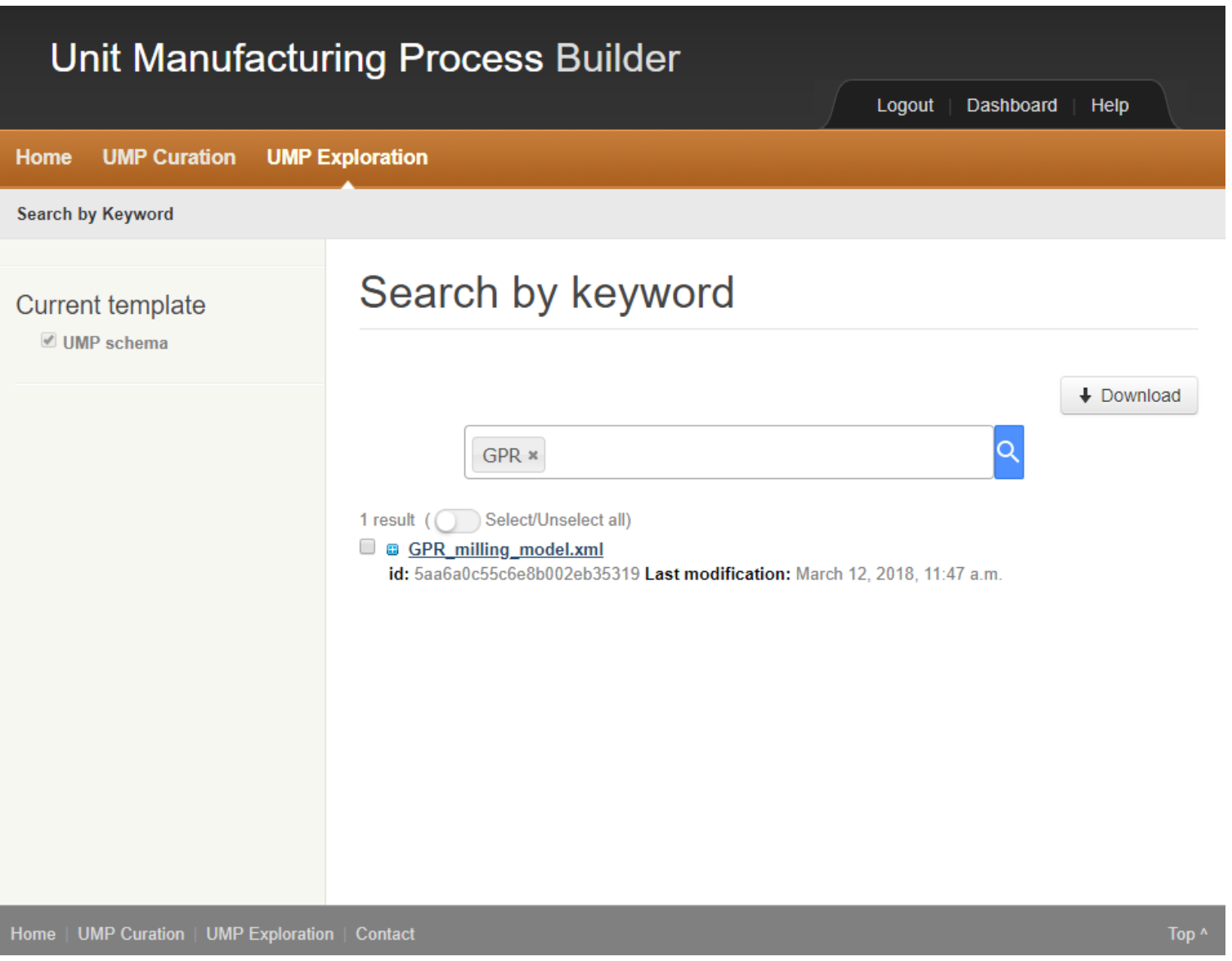

Fig. 21. Result of a Keyword Search

\subsection{Visualization}

After the validation of your model, or when you select a model from the validation page, you are redirected to the visualization page. This page provides an interactive visualization of the UMP model as shown in Figure 22. The graphical representation is similar to the graphical representation proposed in the ASTM E3012 standard.

\subsubsection{Meta-Information}

The graphical representation first includes all the meta-information of the UMP, the authors, the keywords, the model bounds and the possible reviews. Each section is expandable by clicking on the name of the section.

\subsubsection{Main Information}

The fives boxes include the information about the inputs, product and process information, resources, transformations and outputs of the model. Each box is expandable by clicking on 


\section{Unit Manufacturing Process Builder}

\section{Home UMP Curation UMP Exploration}

Search by Keyword

\section{MillingExample.amogh.xml}
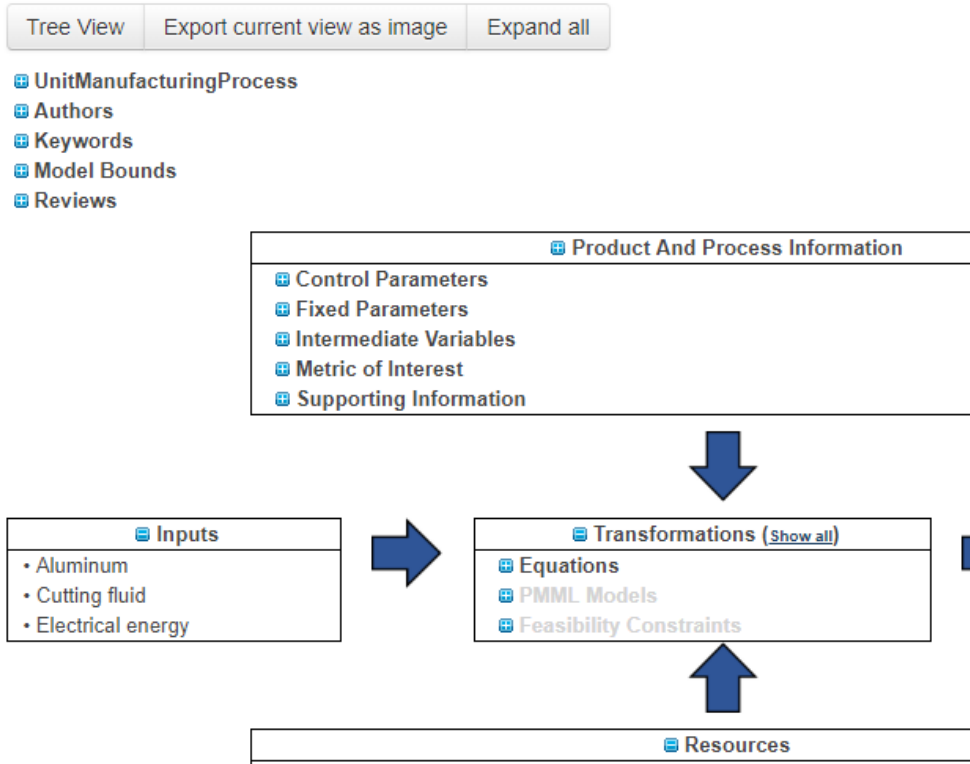

\begin{tabular}{|l|}
\hline \multicolumn{1}{|l|}{ Resources } \\
\hline - Operator \\
- Tool list \\
- Agie Charmilles HPM600U Brochure \\
\hline
\end{tabular}

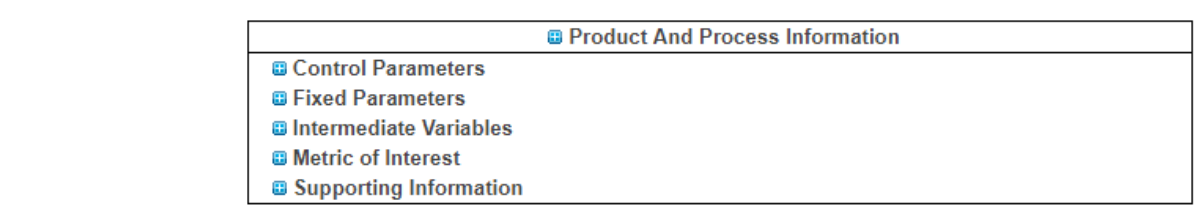

- Agie Charmilles HPM600U Brochure

Fig. 22. Visualization Page

the name of the sub-sections of the boxes. When you click on elements inside the boxes, a pop-up appears and provides any additional information about the given element as shown in Figure 23.

\subsubsection{Full List of Transformations}

At the bottom of the graphical representation, you can obtain the list of all equations included as transformations in the model. 


\section{Unit Manufacturing Process Builder}

Home UMP Curation UMP Exploration Composer

Query by Example Search by Keyword OAI-PMH Search by Keyword

\section{MillingExample.amogh.xml}
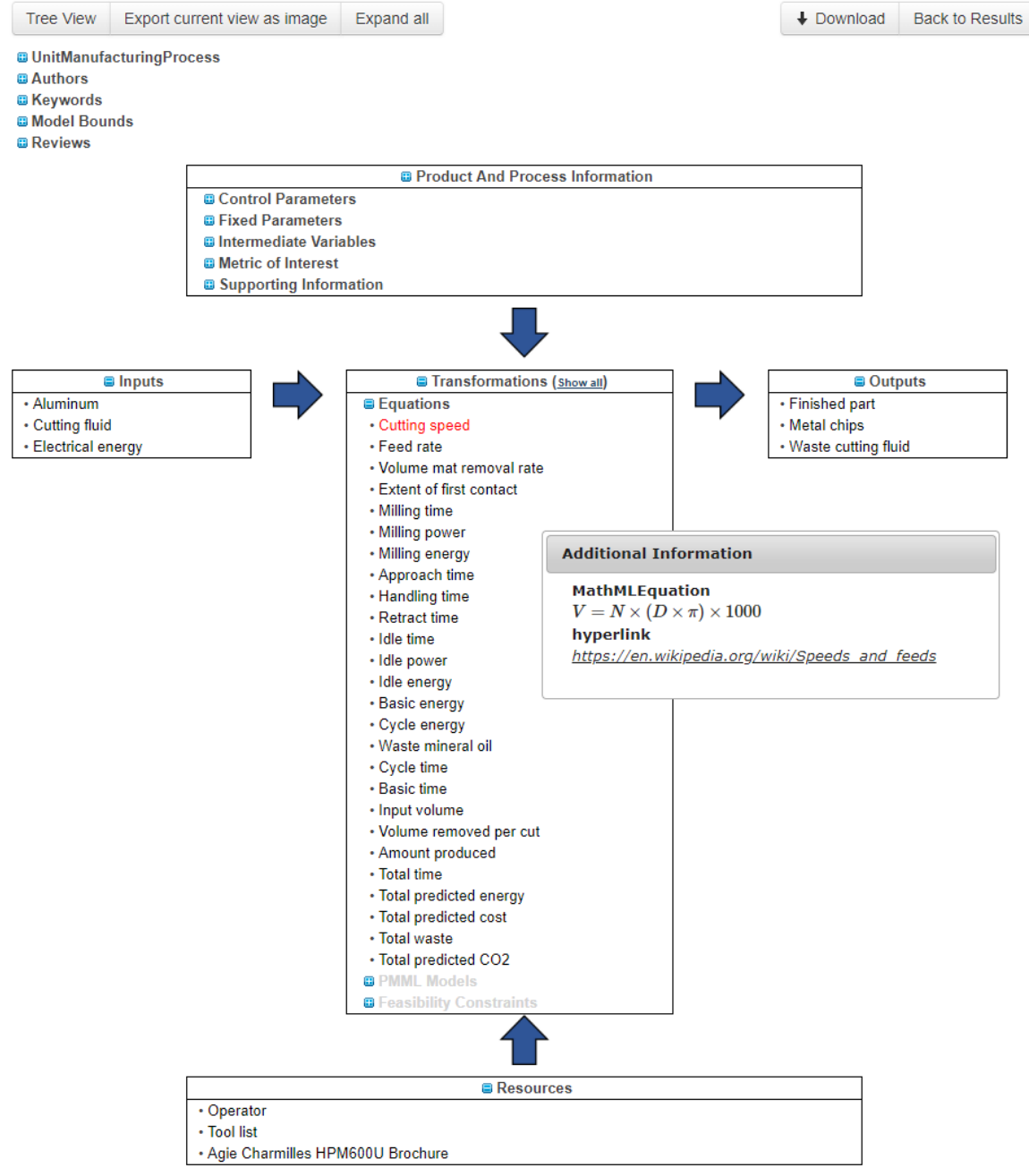

$\boxplus$ Full list of transformations

Fig. 23. Additional Information 


\subsubsection{Additional Features}

The page provides capabilities to visualize the model in a tree view, export the current state of the view as an image, expand all the sections of the representation, and download the model in an xml format by clicking on the buttons on the top of the graphical representation.

\subsection{Dashboard}

The dashboard allows you to edit your profile, change your password, and see the list of submitted models and work-in-progress. To reach the dashboard, press the Dashboard tab from any page.

\subsubsection{My Profile}

When you click on the tab, you are redirected to the My Profile section of the dashboard where you can edit your profile and change your password as shown in Figure 24.

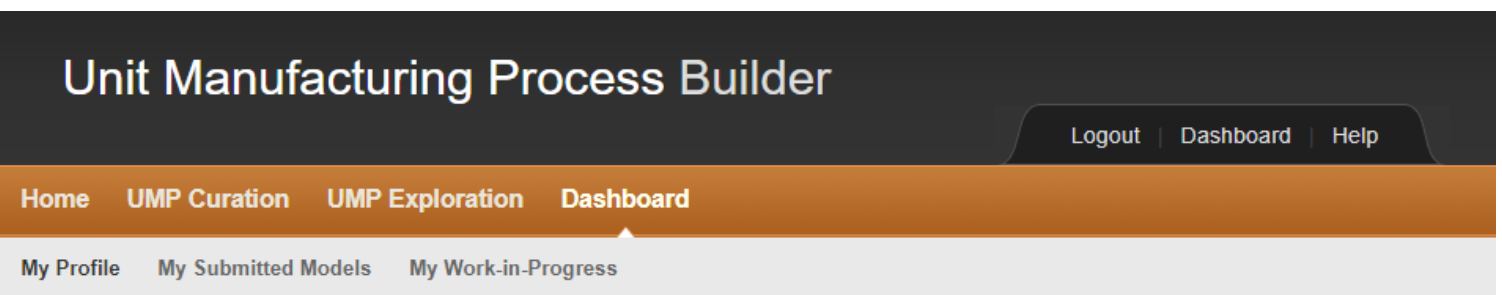

\section{My Profile}

Fig. 24. Profile Page 


\subsubsection{My Submitted Models}

You can also consult the models you have submitted by clicking on the My Submitted Models tab. Figure 25 shows the page displayed to consult the models. You can decide to view a submitted model, change the model name, modify the model, delete the model or change the ownership of the model by clicking on the appropriate buttons.

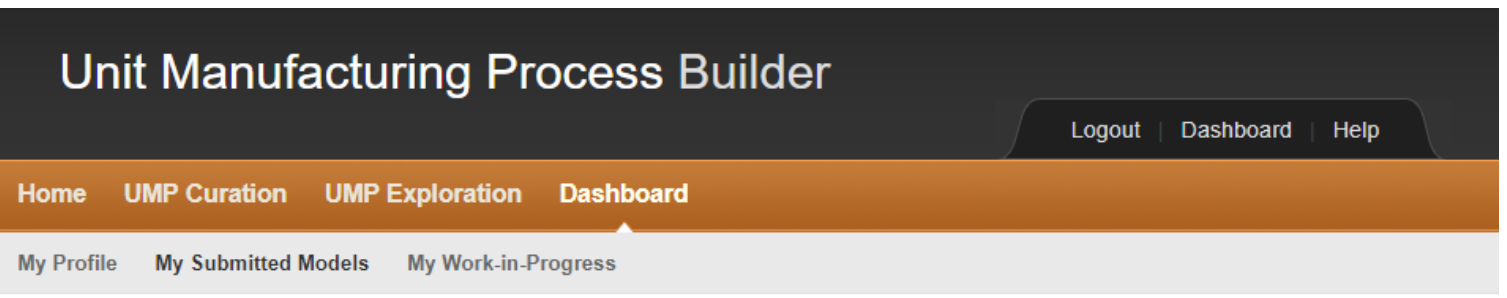

\section{My submitted models}

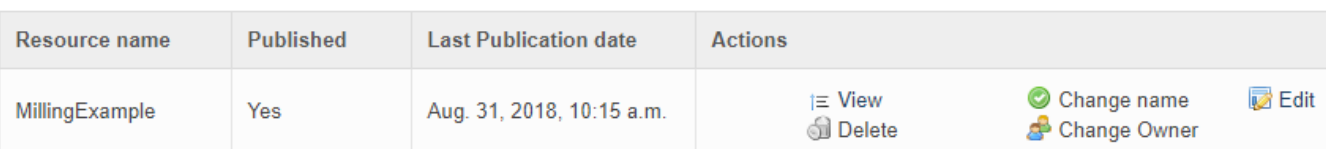

Fig. 25. Submitted Models Page

\subsubsection{My Work-in-Progress}

Your work-in-progress are available by clicking on the My Work-in-Progress tab. You can decide to view a work-in-progress, change the name, continue working on the model, delete the work-in-progress or change the ownership of the work-in-progress by clicking on the appropriate buttons. Figure 26 show the page that displays the different options. 


\section{Unit Manufacturing Process Builder}

\section{Home UMP Curation UMP Exploration Dashboard}

My Profile My Submitted Models My Work-in-Progress

\section{My Work-in-Progress}

You can review all the work-in-progress you have saved. You can either delete your work-in-progress or change the owner.

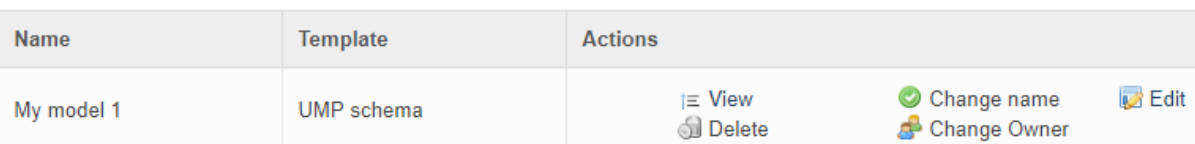

Fig. 26. Work-in-Progress Page

\subsection{Administration}

If you have been providing administration credentials, you have access to the administration section. To reach this section, you need to click on the Administration tab located at the bottom of the home page. You will be redirected to the User Management section of the administration page as shown in Figure 27. 


\section{Unit Manufacturing Process Builder}

\section{Home User Management Templates \& Types Repositories Website OAI-PMH}

Manage Users Account Requests Contact Messages

\section{User Management}

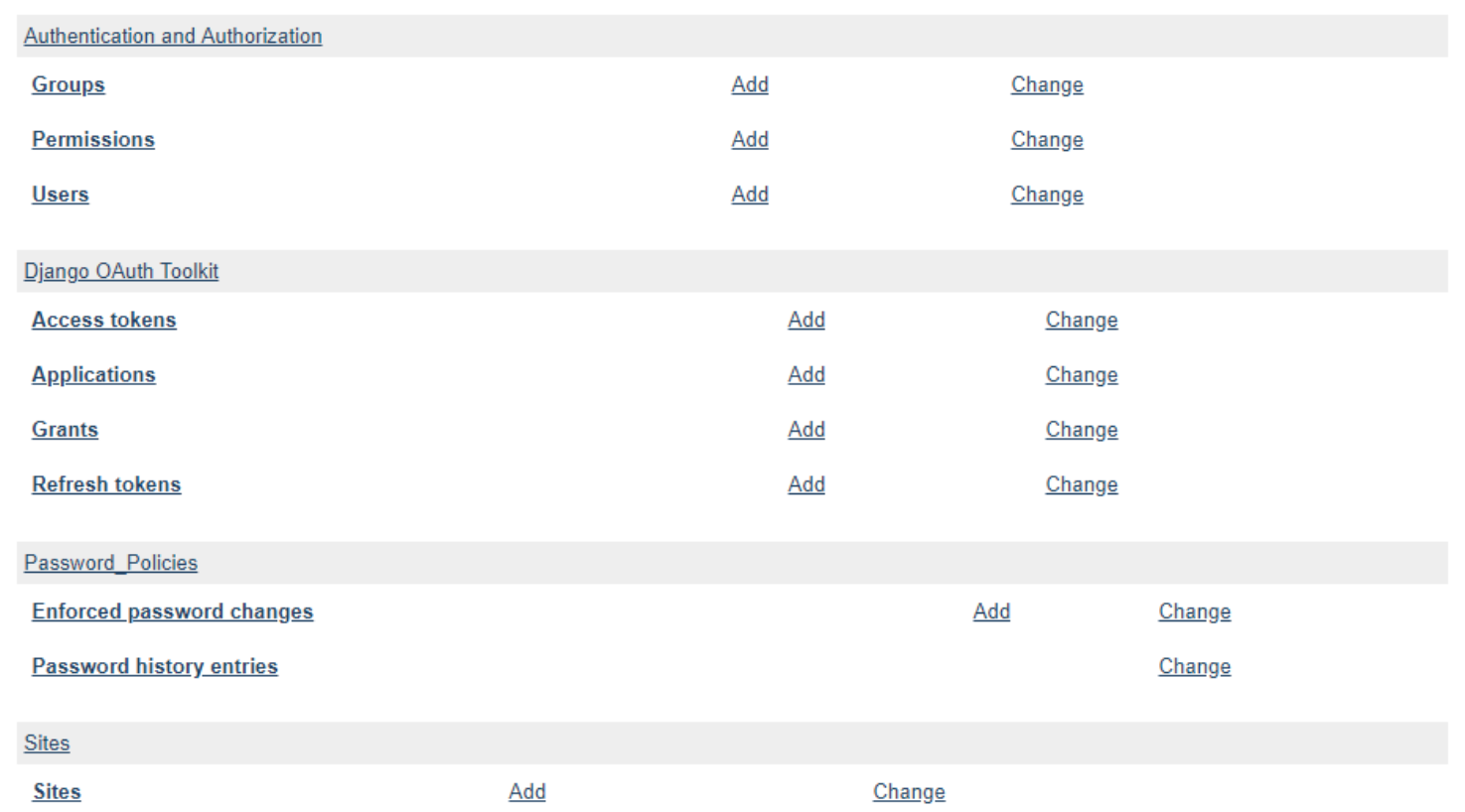

Fig. 27. User Management Page

\subsubsection{Manage Users}

You can manage the users and modify their permissions by clicking on the Change link for Users. You will be redirected to the list of users shown in Figure 28 and you can click on the users you wish to modify.

Once you have selected the user to change, you are redirected to page to make the necessary modification on the user name, personal info, permissions as shown in Figure 29. 


\section{Unit Manufacturing Process Builder}

\section{Home User Management Templates \& Types Repositories Website OAI-PMH}

Manage Users Account Requests Contact Messages

\section{Select user to change}

\begin{tabular}{llll}
$\cdot$ Add user & & \\
\hline
\end{tabular}

Fig. 28. User List

\subsubsection{Manage Groups}

Similarly, you can modify or create a new group by clicking the add or change link for Groups. Groups are used to automatically assign a set of permissions. If you click on the change link, you can then select the group to modify as shown in Figure 30.

You will be redirected to the page to select which permissions to provide to the group you are creating or modifying as shown in Figure 31.

These are the two main features you should manage in the Manage Users section. 


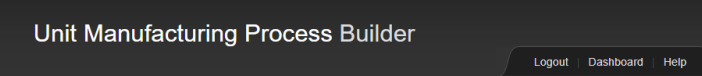

Home User Management Templates \& Types Repositories Website OAl-PMH

Manage Users Account Requests Contact Messages

Change user

Home, Auth, Users, David

History.

Usoname: Dakn

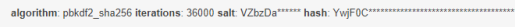

Raw passwords are not tlored, so there is no way to see this user's password, but you can change the passwerd

Personal info

First name: David

Last name

echevalier

Emal addess:

chevallercavid@gmal..com

Permissions

Adive

$\square$ Stati status
Designetes whether the user can log into this s dmin stt

$\square$ Superuser status

ats all permissions whenout explichty yssigning them

Groups: Available groups @

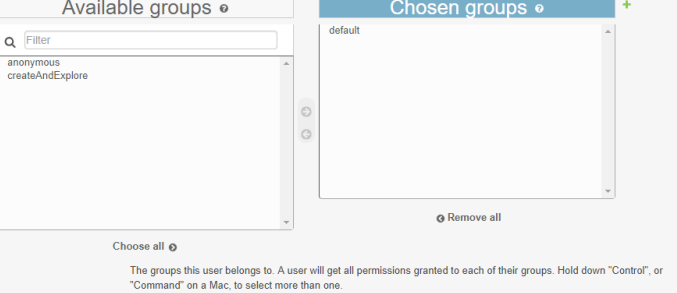

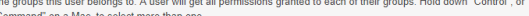

Available user permissions • Chosen user permissions •

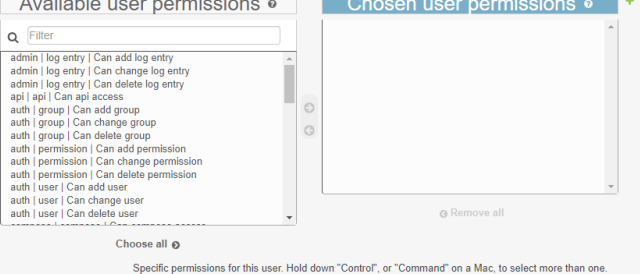

Important dates

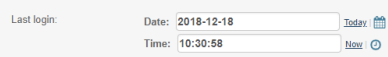

Date joined: Date: $2017 \cdot 11-09$

Time: 10:05:25

SAVEE Save and add another Save and continue edting

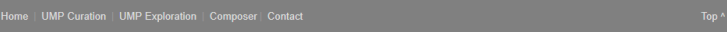

Fig. 29. User Modification 


\section{Unit Manufacturing Process Builder}

\section{Home User Management Templates \& Types Repositories Website OAI-PMH}

Manage Users Account Requests Contact Messages

\section{Select group to change}

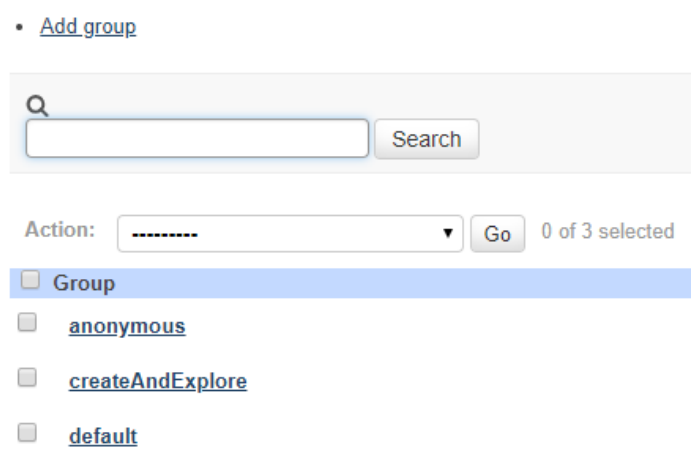

3 groups

Fig. 30. Group List

\subsubsection{Account Requests}

To consult the pending account requests, you can click on the Account Request tab. Figure 32 shows how the requests are displayed. You are be able to Accept or Deny the requests pending for approval. The user who requested the account is notified of your decision by email.

\subsubsection{Contact Messages}

A user can send contact messages that available by clicking on the Contact Messages tab. The list of messages is displayed, and you can then answer to the user by email. 


\section{Unit Manufacturing Process Builder}

\section{Home User Management Templates \& Types Repositories Website OAI-PMH}

Manage Users Account Requests Contact Messages

\section{Change group}

Home , Auth , Groups ; createAndExplore

- History.

Name:

createAndExplore

Permissions:

Available permissions •

\section{Q Filter}

admin | log entry | Can add log entry

admin | log entry | Can change log entry

admin | log entry | Can delete log entry

api | api | Can api access

auth | group | Can add group

auth | group | Can change group

auth | group | Can delete group

auth | permission | Can add permission

auth | permission | Can change permission

auth | permission | Can delete permission

auth | user | Can add user

auth | user | Can change user

auth | user | Can delete user

\section{Choose all 0}

Hold down "Control", or "Command" on a Mac, to select more than one.

\section{Chosen permissions o}

curate | curate | Can curate access

curate | curate | Can curate delete document

curate | curate | Can curate edit document

curate | curate | Can curate view data save repo

explore | explore | Can explore access

explore | explore | Can explore delete query

explore | explore | Can explore save query

Fig. 31. Group Modification

\section{Summary}

In summary, this document described the features and functionality of the UMP Builder for curating and visualizing and querying unit manufacturing process (UMP) models. UMP models are captured in an XML format that is compliant to the ASTM E3012 standard.

The UMP Builder provides features to curate the UMP models into a repository. The 


\section{Unit Manufacturing Process Builder}

\section{Home User Management Templates \& Types Repositories Website OAI-PMH}

Manage Users Account Requests Contact Messages

\section{Account Requests}

\begin{tabular}{|l|l|l|l|l|l|}
\hline User & First Name & Last Name & Email Address & Actions \\
\hline new_user & New & User & lechevalierdavid@gmail.com & O Accept & $\mathbf{X}$ Deny \\
\hline
\end{tabular}

Fig. 32. Account Request List

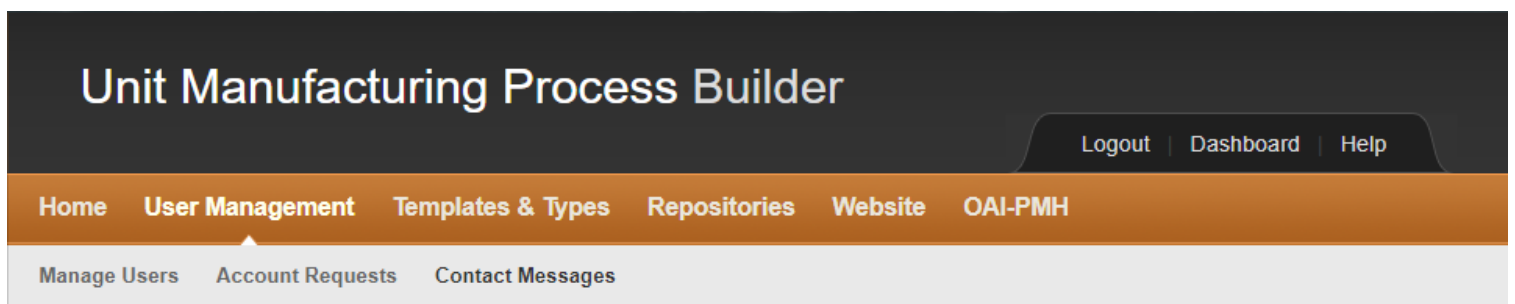

\section{Contact Messages}

\begin{tabular}{|l|l|l|l|}
\hline Name & Email Address & Message & Actions \\
\hline $\begin{array}{l}\text { David } \\
\text { Lechevalier }\end{array}$ & lechevalierdavid@gmail.com & $\begin{array}{l}\text { Hello, I have a question. } \\
\text { David }\end{array}$ & \\
\hline
\end{tabular}

Fig. 33. Contact Messages

tool also provides query capabilities in order to retrieve existing models. Interactive visualizations of the models are available in order to go through all the information collected in a model. The document describes how to build your model section by section, how to submit the models to the repository, and how to visualize models that are recorded in the repos- 
itory. This work is an on-going process in order to facilitate the digitalization of UMPs. Defining a robust schema to represent the UMP is key to enabling this digitalization. A robust application is necessary to increase the adoption of such a process in the manufacturing area and will be the subject of future work and collaboration with partners involved in this area of research.

\section{Disclaimer}

Certain commercial products may have been identified in this paper. These products were used only for demonstration purposes. This use does not imply approval or endorsement by NIST, nor does it imply that these products are necessarily the best for the purpose.

\section{References}

[1] Bernstein WZ, Lechevalier D, Libes D (2018) Ump builder: capturing and exchanging manufacturing models for sustainability. ASME 2018 13th International Manufacturing Science and Engineering Conference (American Society of Mechanical Engineers), , pp V001T05A022-V001T05A022.

[2] Bernstein WZ, Mani M, Lyons KW, Morris K, Johansson B (2016) An open web-based repository for capturing manufacturing process information. ASME 2016 international design engineering technical conferences and computers and information in engineering conference (American Society of Mechanical Engineers), , pp V004T05A028V004T05A028.

[3] Frechette S (2019) Smart manufacturing systems design and analysis program. Available at https://www.nist.gov/programs-projects/ smart-manufacturing-systems-design-and-analysis-program.

[4] Dima A, Bhaskarla S, Becker C, Brady M, Campbell C, Dessauw P, Hanisch R, Kattner U, Kroenlein K, Newrock M, et al. (2016) Informatics infrastructure for the materials genome initiative. Jom 68(8):2053-2064.

[5] Bernstein WZ (2019) A reference schema for the unit manufacturing process information model. Available at https://doi.org/10.18434/M32027.

[6] Bernstein WZ, Lechevalier D (2019) A reference schema for the unit manufacturing process information model. Journal of Research of the National Institute of Standards and Technology 124:1-7.

[7] Ausbrooks R, Buswell S, Carlisle D, Dalmas S, Devitt S, Diaz A, Froumentin M, Hunter R, Ion P, Kohlhase M, et al. (2003) Mathematical markup language (mathml) version 2.0 . w3c recommendation. World Wide Web Consortium 2003. 